\title{
Repetitive EEG recordings are necessary for the diagnosis of early myoclonic encephalopathy
}

\author{
Hamit Özyürek, Güzide Turanli, Didem Aliefendioglu*, Turgay Coskun** \\ Departments of Pediatric, Pediatric Neurology Unit, Hacettepe University Faculty of Medicine, *Ankara Social Security Hospital, \\ Neonatology Unit, ${ }^{*}$ Hacettepe University Faculty of Medicine, Department of Pediatrics, Metabolism and Nutrition Unit, Turkey
}

Early myoclonic encephalopathy (EME) is a rare malignant epileptic syndrome. The erratic myoclonus with or without focal motor seizures, time of onset before 3 months of age, and suppression-burst (SB) pattern in EEG are accepted as the diagnostic criteria for EME. We report a 40-day-old infant with the diagnosis of non-ketotic hyperglycinemia (NKHG). The infant developed myoclonic and focal tonic seizures on the first day of life. His first sleep EEG recorded after onset of seizure was normal. Because of the diagnosis of NKHG and early developed myoclonic seizure, we thought the infant might be EME, and repeated sleep EEG on admission in which asymmetrical SB pattern was seen. We concluded that the absence of SB pattern in the first EEG recording does not exclude the diagnosis of EME, but repetition of EEG is necessary to demonstrate the presence of SB pattern to meet the diagnostic criteria for EME.

Key Words: Early myoclonic encephalopathy, suppressionburst, EEG, non-ketotic hyperglycinemia

Early epileptic encephalopathy with suppression burst (SB) is a malignant epilepsy syndrome seen during the neonatal and early infantile periods. Early infantile epileptic encephalopathy (EIEE or Ohtahara syndrome) and early myoclonic encephalopathy (EME) are included in this epileptic group. ${ }^{[1]}$

According to the International Classification of Epilepsies and Epileptic Syndromes (ILAE, 1989), EME is categorized as age-related, generalized symptomatic epilepsies of non-specific etiology. EME is clinically characterized by the onset of erratic or fragmentary myoclonus. Erratic, partial myoclonus usually appears as the first seizure, even as early as a few hours after birth. The myoclonus usually involves the face or extremities. Neurological abnormalities are constant: very severe delay in psycho-motor acquisitions, marked hypotonia, and disturbed alertness sometimes with vegetative state. In EME, the EEG shows a SB pattern, which consists of bursts lasting 1 to 5 seconds alternating with almost flat periods lasting 3 to 10 seconds. In addition to the clinical features, $\mathrm{SB}$ is accepted as one of the diagnostic criteria of EME..$^{[1-3]}$ Here, we report a 40-day-old infant presenting with EME who had normal EEG initially and an asymmetrical SB pattern in the repeat EEG in asleep state.

\section{Case Report}

A 40-day-old infant was born normally after an uncomplicated term pregnancy. After birth, his mother recognized that the baby was hypotonic. There were difficulties in sucking and swallowing. He developed myoclonic and focal tonic seizures on the first day of life. His asleep EEG recorded within 2 days after seizure onset was normal, and he was put on phenobarbital therapy. At this time, biochemical tests (including calcium, magnesium, glucose) were normal. Metabolic screening showed an elevation in the urinary glycine and glutamine excretion. The serum and CSF levels of glycine were 403 $\mathrm{nmol} / \mathrm{ml}(105-318)$, and $214 \mathrm{nmol} / \mathrm{ml}$ (1.3-11.51) respectively. The findings were consistent with the diagnosis of non-ketotic hyperglycinemia (NKHG). Sodium benzoate and dextromethorphan were added to the treatment. Partial agenesis of the corpus callosum was seen in cranial magnetic resonance imaging. He was subsequently transferred to our center because of uncontrolled seizures. He was the second child of healthy and first-degree consanguineous parents. The first child was lost with same complaints in the neonatal period.

On admission, his vital signs were normal. His weight, length, and head circumference were within normal limits. He was lethargic and hypotonic. EEG was repeated on admission. This EEG revealed that 1-2 seconds of sharp waves and sharply contoured slow waves were followed by voltage attenuation periods lasting 5-10 seconds. Bursts were mostly asynchronous with right hemispheric predominance. Voltage suppression was noted on the left hemisphere, predominantly over the temporal region. Overall tracing showed burst-suppression pattern with marked hemispheric asymmetry and asynchrony [Figure 1]. The phenobarbital dose was increased to $15 \mathrm{mg} / \mathrm{kg}$ daily, and pyridoxal phosphate $(100 \mathrm{mg} / \mathrm{d})$ was added to control seizures. But there was no improvement in clinical and EEG findings. The patient was discharged on high-dose phenobarbital treatment on the eighth hospital day. Two weeks later, the patient was re-admitted because 


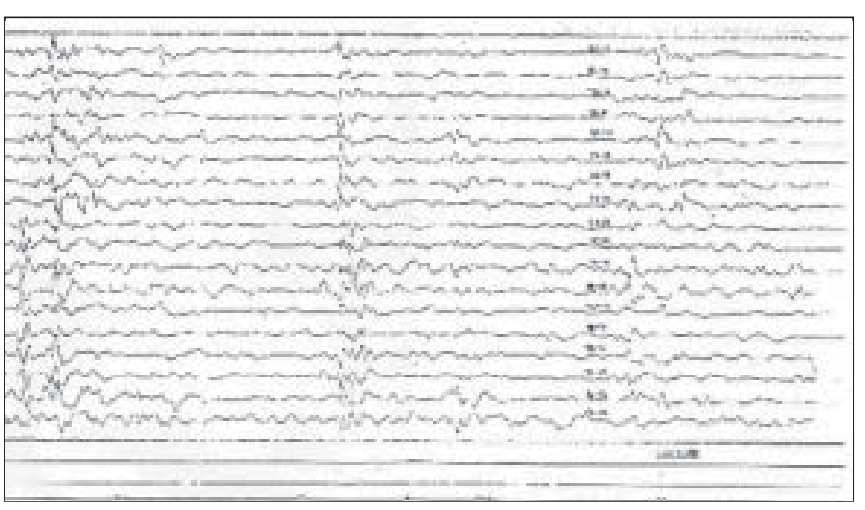

Figure 1: Interictal asymmetric suppression-burst pattern in second asleep EEG

of phenobarbital toxicity. Treatment was changed to a combination of phenobarbital $(5 \mathrm{mg} / \mathrm{kg}$ per day) and vigabatrin $(100 \mathrm{mg} / \mathrm{kg}$ per day). The patient received the combination of phenobarbital and vigabatrin for approximately three months. During this period, no changes were observed in either the seizure frequency or the EEG findings. Then the treatment was substituted with corticotropin. The patient was out of follow-up with parental consent.

\section{Discussion}

Etiologies of EME are heterogeneous. Frequent occurrence of familial cases indicates that an inborn error of metabolism may be the most likely cause of EME. NKHG, propionic aciduria, and D-glyceric acidemia were reported to lead to EME, but there were still many cases with unknown causes. ${ }^{[4,5]}$ In a study on 29 cases fulfilling the EME criteria, there were inborn errors of metabolism in eight, congenital brain malformations in five, and birth asphyxia in six cases. The remaining ten cases were accepted as cryptogenic. ${ }^{[5]}$ Chen et al detected a metabolic disease in 4 out of 5 EME cases. ${ }^{[1]}$

In EME, EEG is characterized by a burst-suppression pattern with bursts of spikes, sharp waves, and slow waves, which are irregularly intermingled and separated by periods of electrical silence. The bursts usually last 1 to 5 seconds with inactive periods of 3 to 10 seconds. The EEG paroxysms may be either synchronous or asynchronous over both hemispheres. The burst phases of SB in EME are lower in amplitude than EIEE. The suppression phases of SB in EME are longer in duration than most cases of EIEE. SB pattern of EME is mainly noted during the sleep state, but that of EIEE is noted in both awake and sleep states. Moreover, SB pattern may be detected in both awake and sleeping states in non-ketotic hypergylcinemia cases. ${ }^{[3]} \mathrm{SB}$ pattern tends to be replaced by atypical hypsarrhythmia or by multifocal paroxysms after 35 months of age. However, in most cases, the appearance of atypical hypsarrhythmia is transient and a subsequent return to SB pattern is observed and thereafter, the SB pattern characteristically persists for a prolonged period. . $^{[1,3,6,7]}$

The asymmetric pattern of burst-suppression activity in our patient could be the result of partial agenesis of the corpus callosum. Agenesis of the corpus callosum is a frequent congenital malformation with many etiologies and clinical features. Although it can occur as an isolated defect which is usually sporadic, it is frequently associated with other malformations, genetic syndromes and metabolic disorders. In a study on 7 patients with agenesis of the corpus callosum, the interhemispheric coherences on resting EEG were lower than in the control group. ${ }^{[8]}$ The results of another study on 4 subjects with agenesis of the corpus callosum were consistent with the latter one. ${ }^{[9]}$

SB pattern is not always found at seizure onset. ${ }^{[4]}$ Sometimes, SB pattern develops after clinical presentation as in our patient. Besides, SB pattern may never be observed in a subgroup of EME cases resulting from NKHG. ${ }^{[4]}$ However, EEG in them shows multiple paroxysmal abnormalities (generalized irregular spike-waves, polyspike-waves and independent focal or multifocal spikes) with randomly asynchronous attenuation. Later in the follow-up, this EEG pattern is replaced directly by hypsarrhythmia. ${ }^{[3,4]}$

Because of its rarity, most of the data about the treatment of seizure in EME cases are obtained from case reports. Conventional antiepileptic drugs, ACTH, corticosteroid and pyridoxine were ineffective to control the seizures. ${ }^{[3]}$ According to the current literature, ACTH, ketogenic diet, liposteroid, oral high-dose phenobarbital, and izonisamid were found to be more beneficial in EIEE than EME. ${ }^{[10-12]}$ We tried to control the seizures with high-dose phenobarbital and pyridoxal phosphate, however we could not observe the benefit of high-dose phenobarbital which we used for a short period because of its side effects.

We added vigabatrine to phenobarbital therapy, because of some promising reports on vigabatrine use. Kalra et al analyzed the infantile epileptic encephalopathies including West syndrome, Lennox-Gastaut syndrome, EME, EIEE with SBs. Vigabatrin was used in eight infantile epileptic encephalopathies patients with good response in $25 \%$, fair response in $25 \%$, and poor response in $50 \%$ of cases. ${ }^{[13]}$ Seizures in a term infant with Ohtahara syndrome, and in a pre-term neonate with similar clinical features failed to respond to conventional anticonvulsants, but were controlled with vigabatrin monotherapy. ${ }^{[14]}$

In conclusion, the absence of SB pattern in the first EEG recording does not exclude the diagnosis of EME, but repetition of EEG over time, also in asleep state, is mandatory because the presence of SB pattern is necessary to satisfy all diagnostic criteria for EME. The asymmetric pattern of SB activities may be related to agenesis of the corpus callosum as in our patient.

\section{References}

1. Chen PT, Young C, Lee WT, Wang PJ, Peng SS, Shen YZ. Early epileptic encephalopathy with suppression burst electroencephalographic pattern- an analysis of eight Taiwanase patients. Brain Dev 2001;23:715-20.

2. Commission on Classification and Terminology of International League Against 
Epilepsy: Proposal for revised Classification of Epilepsies and Epileptic syndromes. Epilepsia 1989;30:389-99.

3. Ohtahara S, Ohtsuka Y, Oka E. Epileptic encephalopathies in early infancy. Indian .J Pediatr 1997;64:603-12.

4. Wang PJ, Lee WT, Hwu WH, Young C, Yau KT, Shen YZ. The contraversy regarding diagnostic criteria for early myoclonic encephalopathy. Brain Dev $1998 ; 20: 530-5$.

5. Lombroso CT. Early myoclonic encephalopathy, early infantile epileptic encephalopathy, benign and severe infantile myoclonic epilepsies: A critical review and personal contributions. J Clin Neurophysiol 1990;7:380-408.

6. Ohtahara S, Ohtsuka Y, Erba G. Early epileptic encephalopathy with suppression burst. In: Engel J, Pedley TA, editors. Epilepsy: A Comprehensive Text book. Volume III. Philadelphia: Lippincott-Raven Publishers; 1997. p. 2257 61.

7. Dalla BB, Dulac O, Fejerman N, Dravet C, Capovilla G, Bondavalli S, et al. Early myoclonic epileptic encephalopathy (E.M.E.E.). Eur J Pediatr $1983 ; 140: 248-52$.

8. Koeda T, Knyazeva M, Njiokiktjien C, Jonkman E.J, De Sonneville L, Vildavsky $\mathrm{V}$. The EEE in acallosal children. Coherence values in the resting state: Left hemisphere compensatory mechanism? Electroencephalogr Clin Neurophysiol
$1995 ; 95: 397-407$

9. Nielsen T, Montplaisir J, Lassonde M. Decreased interhemispheric EEG coherence during sleep in agenesis of the corpus callosum. Eur Neurol 1993;33:173-6.

10. Ohtahara S, Ohtsuka Y, Yamatogi Y, Oka E, Inoue E. Early-infantile epileptic encephalopathy with suppression-bursts. In: Roger J, Bureau M, Dravet CH Dreifuss FE, Perret A, Wolf P, editors. Epileptic syndromes in infancy, childhood, and adolescence, $2^{\text {nd }}$ Ed. London: John Libbey; 1992. p. 25-34.

11. Ozawa H, Kawada Y, Noma S, Sugai K. Oral high-dose phenobarbital therapy for early infantile epileptic encephalopathy. Pediatr Neurol 2002;26:222-4.

12. Ohno M, Shimotsuji Y, Abe J, Shimada M, Tamiya H. Zonisamide treatment of early infantile epileptic encephalopathy. Pediatr Neurol 2000;23:341-4.

13. Kalra V, Gulati S, Pandey RM, Menon S. West syndrome and other infantile epileptic encephalopathies- Indian hospital experience. Brain Dev 2002;24:1309.

14. Baxter PS, Gardner-Medvin D, Barwick DD, Ince P, Livingston J, MurdochEaton D. Vigabatrin monotheraphy in resistant neonatal seizures. Seizure $1995 ; 4: 57-9$.

Accepted on 22-10-2003 